\title{
MassARRAY-based single nucleotide polymorphism analysis in breast cancer of north Indian population
}

\author{
Divya Bakshi", Ashna Nagpal', Varun Sharma², Indu Sharma², Ruchi Shah¹, Bhanu Sharma', Amrita Bhat ${ }^{1}$, \\ Sonali Verma', Gh. Rasool Bhat ${ }^{1}$, Deepak Abrol ${ }^{3}$, Rahul Sharma ${ }^{4}$, Samantha Vaishnavi ${ }^{5}$ and Rakesh Kumar ${ }^{1 *}$
}

\begin{abstract}
Background: Breast Cancer (BC) is associated with inherited gene mutations. High throughput genotyping of $B C$ samples has led to the identification and characterization of biomarkers for the diagnosis of BC. The most common genetic variants studied are SNPs (Single Nucleotide Polymorphisms) that determine susceptibility to an array of diseases thus serving as a potential tool for identifying the underlying causes of breast carcinogenesis.

Methods: SNP genotyping employing the Agena MassARRAY offers a robust, sensitive, cost-effective method to assess multiple SNPs and samples simultaneously. In this present study, we analyzed 15 SNPs of 14 genes in 550 samples (150 cases and 400 controls). We identified four SNPs of genes TCF21, SLC19A1, DCC, and ERCC1 showing significant association with $\mathrm{BC}$ in the population under study.

Results: The SNPs were rs12190287 (TCF21) having OR 1.713 (1.08-2.716 at 95\% Cl) p-value 0.022 (dominant), rs1051266 (SLC19A1) having OR 3.461 (2.136-5.609 at 95\% Cl) p-value 0.000000466 (dominant), rs2229080 (DCC) having OR 0.6867 (0.5123-0.9205 at 95\% Cl) p-value 0.0116 (allelic) and rs2298881 (ERCC1) having OR 0.669 (0.46-0.973 at 95\% $\mathrm{Cl}$ ), p-value 0.035 (additive) respectively. The in-silico analysis was further used to fortify the above findings.

Conclusion: It is further anticipated that the variants should be evaluated in other population groups that may aid in understanding the genetic complexity and bridge the missing heritability.
\end{abstract}

Keywords: Breast cancer, Single nucleotide polymorphism, Jammu and Kashmir

\section{Background}

Cancer is a neoplastic disease consisting of cancer cells that harbor numerous biological capabilities that occur due to the accumulation of various genetic aberrations and genomic alterations [1]. In 2018, about 18 million new cases of cancer were recorded and about 9.5 million deaths occurred with lung and $\mathrm{BC}$; being the leading cause of mortality in men and women, respectively [2]. $\mathrm{BC}$ is the leading cancer of women in India, with $27.7 \%$

\footnotetext{
*Correspondence: divya.bakshi09@gmail.com; drrakeshthusoo@gmail.com ${ }^{1}$ School of Biotechnology, Shri Mata Vaishno Devi University, Katra, Jammu and Kashmir, India

Full list of author information is available at the end of the article
}

of all the cancers in women [3]. A study conducted in the Kashmir province of Jammu and Kashmir highlighted $\mathrm{BC}$ to be the second most common cancer in women with $16.1 \%$ of all the cancers, closely following colorectal cancer (16.8\%) [4]. Despite the exacerbating rate of $\mathrm{BC}$ in $\mathrm{J} \& \mathrm{~K}$, there is a high susceptibility of postmenopausal women to develop breast cancer, especially with numerous factors underplay [5, 6]. Early menarche and late menopause ensure a longer exposure to the hormone estrogen thus increasing the risk of $\mathrm{BC}$ [7]. Various studies have identified the variations in high penetrant genes like BRCA1, BRCA2, PTEN, TP53, $C D H 1$, and STK11 along with moderate penetrant genes

C C The Author(s). 2020 Open Access This article is licensed under a Creative Commons Attribution 4.0 International License, which permits use, sharing, adaptation, distribution and reproduction in any medium or format, as long as you give appropriate credit to the original author(s) and the source, provide a link to the Creative Commons licence, and indicate if changes were made. The images or other third party material in this article are included in the article's Creative Commons licence, unless indicated otherwise in a credit line to the material. If material is not included in the article's Creative Commons licence and your intended use is not permitted by statutory regulation or exceeds the permitted use, you will need to obtain permission directly from the copyright holder. To view a copy of this licence, visit http://creativecommons.org/licenses/by/4.0/. The Creative Commons Public Domain Dedication waiver (http://creativecommons.org/publicdomain/zero/1.0/) applies to the data made available in this article, unless otherwise stated in a credit line to the data. 
such as CHEK2, BRIP1, ATM and PALB2 [8, 9] and their association with $B C$. There are about 182 loci that have been identified and are susceptible to BC [10] which accounts for $30 \%$ of the genetic heritability of $\mathrm{BC}$ [11]. Keeping in view the missing heritability and scanty literature of $\mathrm{BC}$ in studied population group, we investigated cancer-associated variants $[12,13]$ to get an insight into their role in $\mathrm{BC}$ development among the population of Jammu and Kashmir. With an effort to bridge this gap, we conducted a case-control association study in population of post-menopausal women from North India.

\section{Methods}

\section{Sample collection and DNA isolation}

Sampling was carried out from January 2015 to December 2018. A total of 550 age-matched samples (150 cases and 400 controls) were recruited for the study. The cases were histo-pathologically confirmed with no evident history of any other cancer. The Institutional Ethical Review Board (IERB) (SMVDU/IERB/18/70) of Shri Mata Vaishno Devi University approved this study. After written informed consent, blood samples, about $2 \mathrm{ml}$ of venous blood was collected into EDTA vacutainers from patients and healthy controls (age and ethnicity matched). Qiagen DNA isolation kit (Catalogue number 51206) was employed for the genomic DNA isolation from a whole blood sample, using the manufacturer's protocol. Quality check was performed using $0.8 \%$ gel electrophoresis and was quantified using the Eppendorf India Pvt. Ltd. Bio-spectrophotometer ${ }^{\mathrm{rm}}$.

\section{SNP genotyping}

Agena Bioscience MassARRAY iPLEX GOLD chemistry technology was used to perform genotyping. Primers flanking the gene region of the SNPs were designed in the AgenaCx platform (GRCh38/hg38) (https://agenacx. $\mathrm{com} /$ ). DNA sample with a concentration of $10 \mathrm{ng} / \mu \mathrm{l}$ was used with volume $1 \mu \mathrm{l}$ for the PCR reaction. The first PCR was performed using forward and reverse primer pool and shrimp alkaline phosphatase was then used to neutralize unincorporated dNTPs from the first PCR reaction. iPLEX extension reaction was performed using pool single base extension primers. Desalting of the amplified products was performed using SpectroCLEAN resin following the protocol [14, 15]. RS100 nanodispenser was used to transfer the cleaned extension products from 384 muti-titration PCR plate to 384 SpectroCHIP and the chip was placed in Agena MassARRAY compact mass spectrometer. A spectrum of the different products was acquired by SpectroAcquire software. Data in the form of genotypes and call clusters were obtained using Agena MassARRAY Typer V4.0.5.
The representative workflow of genotyping has been summarized in Fig. S1 (Supplementary Figure).

\section{Candidate gene and SNP selection}

The genes selected for the present study are involved in following cancer-related pathways: Tumor suppressor, DNA damage and repair, regulation of cell proliferation, telomere maintenance, cell cycle regulation, and apoptosis. Variations with a potential functional effect like causing amino acid alteration, present in the promoter and UTR region, having an effect on splicing site and transcription binding sites were also selected for the study. The potential genetic variants were retrieved from the database. Variations with an allelic frequency greater than $5 \%$ in Gujarati Indians from Houston (GIH) and Western European Ancestry (CEU) as it has been observed that genetic makeup of the studied population is similar to Europeans [16]. The studied genes and variants with their putative role and annotations are summarized in the Table S3.

\section{Statistical analysis}

Chi-square analysis was performed by using the Plink V.1.09 to observe the allelic association between cases and controls [17]. Further, the association analysis was performed with different genetic models, and the association was calculated in terms of Odds Ratio (OR) at 95\% confidence interval (CI) using Statistical Package for the Social Sciences (SPSS) version 20. The corrected OR was obtained using unconditional logistic regression analysis with age and, BMI as confounding factors. Further, the prediction of mRNA secondary structure of the variants have been illustrated by RNA fold [18] and minimum free energy (MFE) calculated from RNA fold was used to measure the stability of the secondary structures of mRNAs. The gene-gene interaction was performed using Cytoscape version 3.8.0 [19] by installing the GeneMANIA plugin [20]. The allele frequencies were compared with the global database 1000 genome [21] using a webbased application LD link [22] to observe the genetic heterogeneity in allele frequencies among different population groups.

\section{Results}

Genetic variants associated with the increased risk of $B C$ in the population under study

Out of the fifteen variants shortlisted four variants namely rs1051266, rs12190287, rs2229080, and rs2298881 in SLC19A1, TCF21, DCC, and ERCC1 genes respectively, were found to be significantly associated with BC in the studied cohort. The variant rs1051266 is located in the second exon of the gene SLC19A1 and is a missense variant. The variant shows a significant association with BC with OR 1.745 (1.321-2.304 at 95\% CI) 
and has a $p$ value $=7.98 \mathrm{E}-05$ (Allelic). A significant association of the variant was also observed in the dominant model with OR $3.461(2.136-5.609$ at $95 \% \mathrm{CI})$ and $\mathrm{p}$ value $=0.000000466$ (Table 1). The variant was providing risk for $\mathrm{BC}$ in the studied cohort. The variant rs12190287 is a 3'UTR variant located in the third exon of the gene TCF21. The allelic association of the variant showed a weak association with $\mathrm{BC}$ and OR observed was $1.306(0.995-1.713$ at $95 \% \mathrm{CI})$ having $p$-value $=$ 0.0491 (Allelic) to observe the maximum effect of allele $\mathrm{C}$, the dominant model was evaluated. Interestingly, the OR observed was $1.713(1.08-2.716$ at $95 \% \mathrm{CI})$ and $p$ value $=0.022$ (Table S2). The variant was providing risk for $\mathrm{BC}$ in the dominant model in the studied cohort. The variant rs2298881 is located at the intron of the ERCC1 gene. Variant rs2298881 was found significantly associated with $\mathrm{BC}$ and the OR observed was 0.6981 $(0.36-0.71$ at $95 \% \mathrm{CI})$ and $\mathrm{p}$ value $=0.01169$ (Allelic). A significant association was observed with the additive model with OR $0.669(0.46-0.973$ at $95 \% \mathrm{CI}), p$-value $=$ 0.035 (Table S2). The variant rs2229080 is a missense variant, located on the third exon of the $D C C$ gene. The variant rs2229080 showed protection against $\mathrm{BC}$ having OR $0.6867(0.5123-0.9205$ at $95 \% \mathrm{CI})$ and $p$ value $=$ 0.011 (Allelic). However non-significant association was observed for rs2229080 using the dominant model: OR $0.797(0.517-1.229$ at $95 \% \mathrm{CI}), p$-value $=0.305$, recessive model: OR $0.505(0.252-1.014$ at $95 \% \mathrm{CI})$, $\mathrm{p}$ value $=0.05$ and Additive model: OR $0.758(0.551-1.042$ at $95 \%$ pvalue $=0.088$ (Table S2).

\section{Genetic variants not associated with $B C$ in the population under study}

The intronic variant rs249954 on the PALB2 gene has been found associated with the breast cancer risk in Chinese population, however has no association with breast cancer in the population under study [23]. The variant rs664677 in the DNA damage response gene $A T M$ though not associated in our population, previously has been reported to be associated with breast cancer and lung cancer risk in Asian people [24]. Another variant rs2981582, in the FGFR2 gene, which was not associated in the population under study, was found associated with breast cancer in Asian and Caucasian population groups [25]. The gene SLC4A7 having the rs4973768 although not significantly associated with breast cancer in our population previously has been found associated with increased risk of breast cancer in Chinese population [26]. The variant rs2363956 of the $A N K L E-1$ gene has been previously reported to be associated with breast cancer risk in Chinese population [27], however its role in the population under study remains ambiguous since only variants with call rate over 90\% were acknowledged in this study. The gene CYP19A1 with the variant rs10046 has previously been reported to be associated with estradiol levels and postmenopausal breast cancer in European population [28]. The 2 variants in TERT gene, rs2736100 and rs2735940, have been reported to be associated with multiple cancers (including breast etc.) in Asian and Caucasian population [29] and lung cancer risk [30] especially in Caucasian population, respectively. The variant rs2975843 in TERF1 gene was not found associated with breast cancer in the population under study however has been reported to be associated with colorectal cancer in European descent population [31]. Studies have shown the BRIP1 gene variant rs4986764 associated with breast cancer in Chinese population [32], however no significant association was found in the studied population. The variant rs3792152 in REV1 gene, not associated with population under study, has been previously reported to be associated with the development of epithelial ovarian cancer in European population [33].

\section{Prediction of mRNA secondary structure}

MFE (Minimum free energy) secondary structure and the centroid secondary structure of the variants were studied (Fig. 1). The secondary RNA secondary structures of rs2229080, rs10,51,266, and rs2298881 polymorphisms revealed a slight variation in the energy of the wild type allele in comparison to the variant type. The variant rs1051266 had MFE of -461.1 ( $\mathrm{Kcal} / \mathrm{Mol})$ for

Table 1 Logistic regression analysis of the variants in the studied population group

\begin{tabular}{|c|c|c|c|c|c|c|c|}
\hline S.No. & GENE & SNP & CASES & CONTROL & H.W.E. & OR AT $95 \% \mathrm{Cl}$ & p-VALUE \\
\hline \multirow[t]{2}{*}{1} & TCF21 & rs12190287 & $C=0.45$ & $C=0.39$ & 0.456 & 1.306 (0.995-1.713) & 0.0491 \\
\hline & & & $G=0.55$ & $G=0.61$ & & & \\
\hline \multirow[t]{2}{*}{2} & $D C C$ & rs2229080 & $C=0.38$ & $C=0.47$ & 0.111 & $0.6867(0.5123-0.9205)$ & 0.01169 \\
\hline & & & $G=0.62$ & $G=0.53$ & & & \\
\hline \multirow[t]{2}{*}{3} & SLC19A1 & rs1051266 & $T=0.46$ & $\mathrm{~T}=0.33$ & 0.053 & $1.745(1.321-2.304)$ & $7.98 \mathrm{E}-05$ \\
\hline & & & $C=0.54$ & $C=0.66$ & & & \\
\hline \multirow[t]{2}{*}{4} & ERCC1 & rs2298881 & $A=0.21$ & $A=0.27$ & 0.124 & $0.6981(0.36-0.71)$ & 0.03043 \\
\hline & & & $C=0.79$ & $C=0.73$ & & & \\
\hline
\end{tabular}




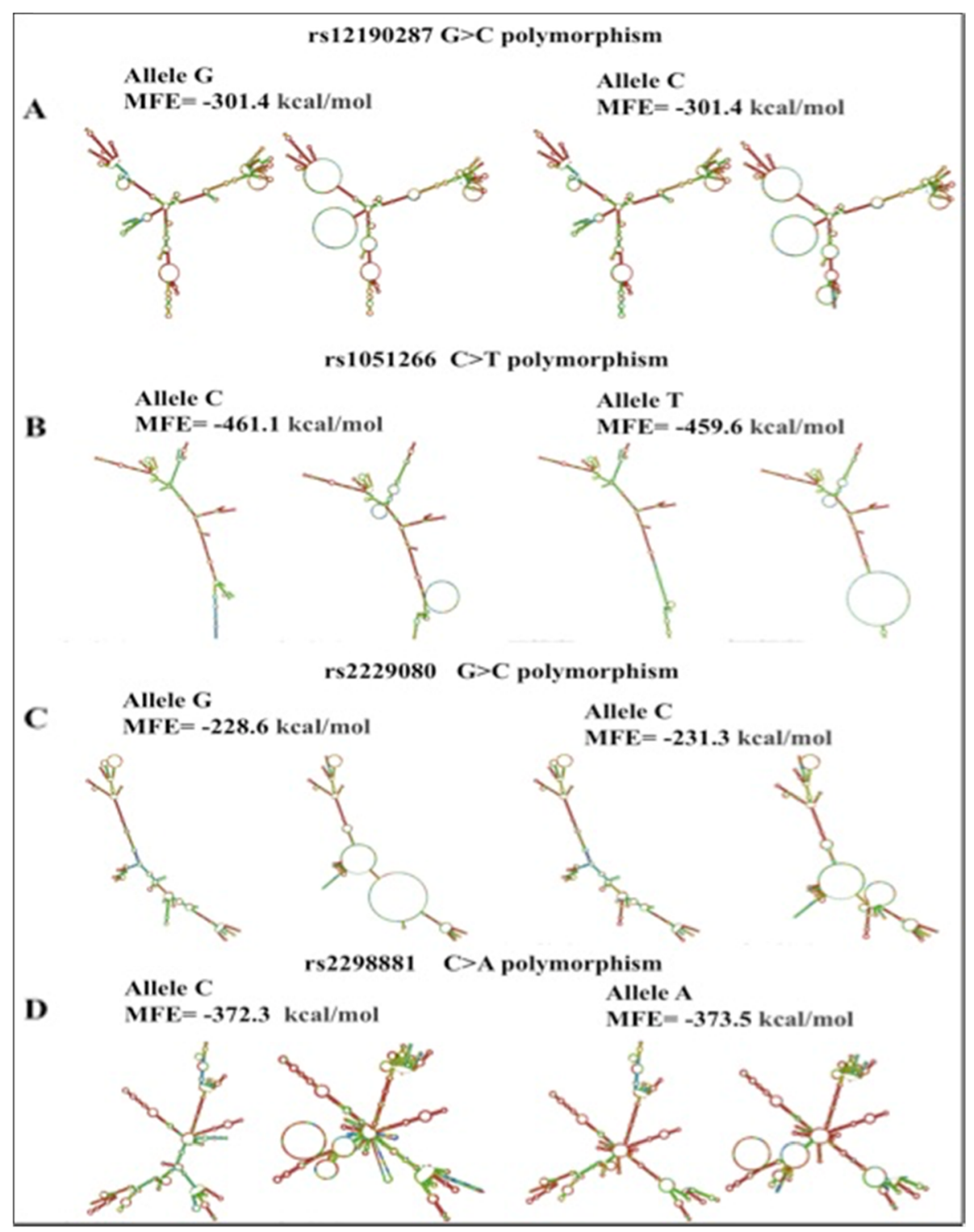

Fig. 1 The predicted MFE and MFE centroid secondary structures of RNA and energies that were calculated by using RNA fold

the ancestral allele $\mathrm{C}$, however, we observed an elevation in the MFE for the altered allele $\mathrm{T}$ being at -459.6 (Kcal/Mol). Also, there was an increase in the MFE of centroid structure for this variant from - 372.57(Kcal/ $\mathrm{Mol})$ for allele $\mathrm{C}$ to -372.57 ( $\mathrm{Kcal} / \mathrm{Mol})$. For the variant rs12190287, no change was observed in the MFE value of the ancestral (G) and altered allele (C) structures, although there was an increase in the MFE of the centroid structure from -234.28 (G) to -222.46 (C) (Kcal/Mol). The variant rs2298881 was showing a decrease in the MFE from - 372.3(Kcal/Mol) for ancestral allele C to $373.5(\mathrm{Kcal} / \mathrm{Mol})$ for altered allele A. The MFE of the centroid structure was also found to be less for the wild allele $\mathrm{C}$ at $-277.15(\mathrm{Kcal} / \mathrm{Mol})$ than the altered allele A at -308.95 (Kcal/Mol). A significant change in the MFE value was observed for the variant rs2229080, the wild allele $\mathrm{G}$ being at $-228.6(\mathrm{Kcal} / \mathrm{Mol})$, and $231.1(\mathrm{Kcal} /$ $\mathrm{Mol}$ ) for the altered allele $\mathrm{C}$. Their centroid structure MFE values also followed the same pattern and varied from being - $141.7(\mathrm{Kcal} / \mathrm{Mol})$ to $-164.3(\mathrm{Kcal} / \mathrm{Mol})$ for the wild and altered allele respectively. The decreased free energy of the wild allele correlates with increased stability in the structure. The observed decrease in MFE of the wild type allele for the variants rs1051266 and the MFE of the centroid structure for the variant rs12190287 corresponds with the increased stability of the wild type allele in both the cases. The altered allele of both the variants has been observed to risk causing. 
The decrease in the stability of the altered structure might be a potent factor posing the risk threat. The altered alleles of the variants rs2298881 and rs2229080 have a low MFE than the wild allele and have been shown for protecting the $\mathrm{BC}$. The low MFE of the altered alleles confers greater stability to these structures than the wild alleles with a higher MFE value. The free energy values have been summarized in the Table S4.

\section{Network analysis}

The potential involvement of DCC1, ERCC1, SLC19A1, and TCF21 relevant genes by querying the genes in the GeneMANIA [20] (Fig. 2). This showed that the expression of the genes is correlated with that of $D C C$. Further network analysis revealed that the $D C C$ gene displays protein-protein interaction with CASP9 that is associated with multiple cancer risks [34]. An interaction, however meek, is seen among the $D C C$ interacting proteins and $T C F 21$. No associated network of interacting proteins was found interacting with SLC19A1.

\section{Discussion}

A replicative case-control study was done in about 550 samples to analyze the variants using Agena MassARRAY genotyping for the population of Jammu \& Kashmir. Here, we investigate various $\mathrm{BC}$ loci in cases and controls. We investigated using the Agena massARRAY platform and identified numerous SNPs that were found significantly associated with $\mathrm{BC}$ genome-wide and independent of each other. Our study demonstrated 4 genome-wide loci which have been associated with $\mathrm{BC}$ development in the population under study. The rsIDs rs12190287 and rs1051266 associated with the genes TCF21 and SCL19A1 are causing risk in our population group. We also found six variants following HWE however not showing significance with $\mathrm{BC}$ development. The allele frequency of all the variants is shown in Figs. S2-S11.

The variant rs1051266 is located on the SLC19A1 gene. SLC19A1 or Solute Carrier family member protein is a gene implicated in placental carcinomas and pediatrics osteosarcomas. Studies have shown the SLC19A1 gene variants to be associate with BC risk in

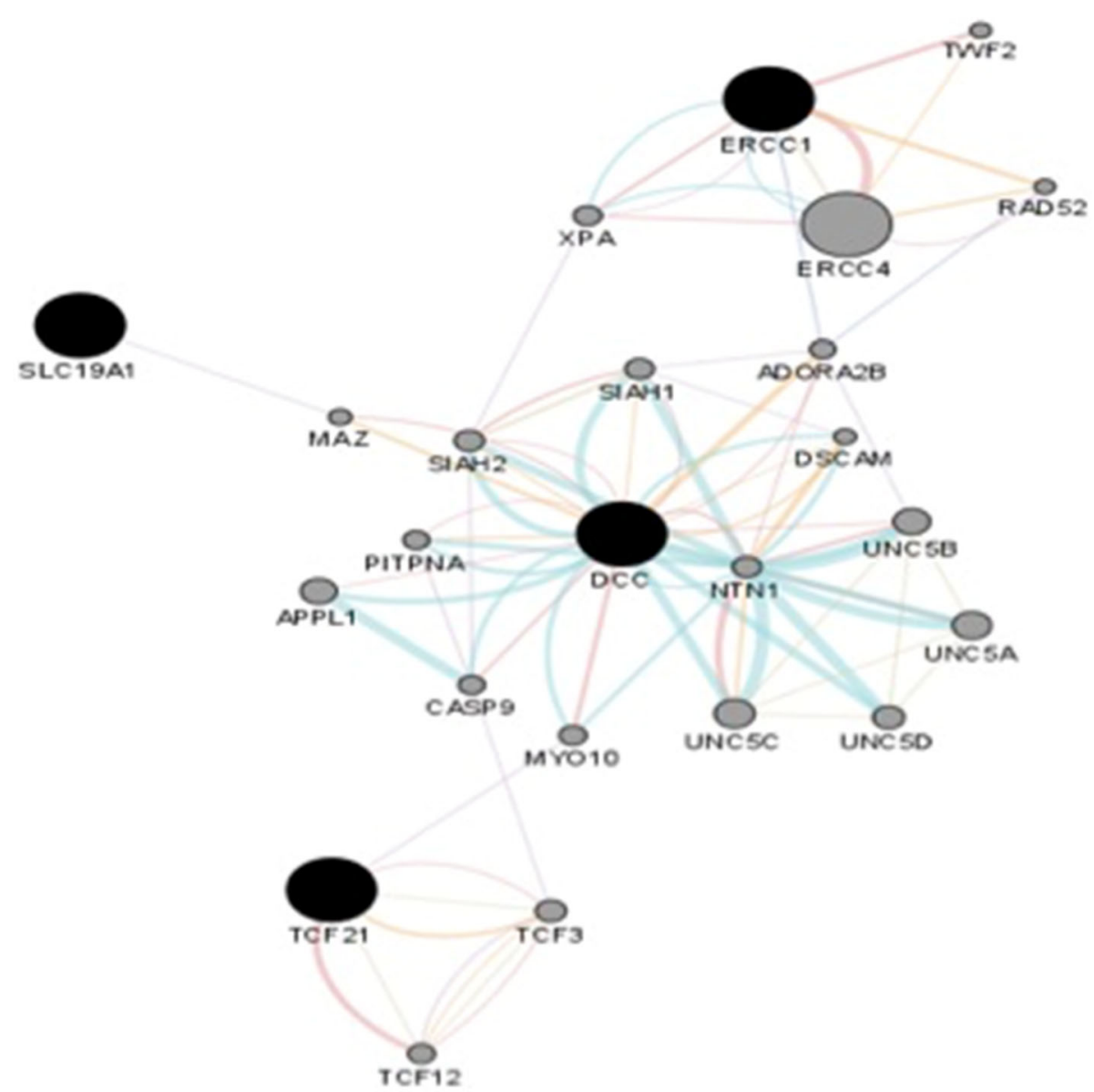

Fig. 2 A network analysis of the associated genes using GeneMANIA database 
worldwide populations [35] including African American women [36]. Our data revealed the variant rs1051266 to be significantly associated with $\mathrm{BC}$ risk in the population under study. Further, the bioinformatic analysis revealed that the associated variants are conserved in primates including humans and have been located in the conserved domain region (Fig. S13). We also studied the genotype tissue expression of the variants (GTEx) with their NES (Normalized Effect Size) values, which have been shown in Table S3. The GTEx with NES (Normalized Effect Size) was used to study the correlation between the genetic variation and gene expression in the human tissues. The variant 1,051,266 (SLC19A1) was significantly showing expression in breast tissue with an NES value of -0.4333 and a $p$-value of $2.4 \mathrm{e}-6(<0.05)$.

TCF21 or Transcription factor gene is a tumor suppressor gene and is associated with Uterine Corpus carcinoma and Pericoronitis. TCF21 is found mutated in several types of cancers [37] Studies have shown a lower expression of TCF 21 in breast tumor tissues corresponding to enhanced tumor size and increased lymph node metastasis [38]. We analyzed the variant rs12190287 $\mathrm{G}>\mathrm{C}$ of the TCF21 gene and found it to be significantly associated with $\mathrm{BC}$ in the studied population group. The variant was found causing risk for $\mathrm{BC}$ in the population. The variant rs12190287 (TCF21) showed significant expression in breast tissue, with an NES value of 0.210 and a $p$-value of $3.3 \mathrm{e}-5$. The positive NES value indicated the up-regulated expression in the breast tissue.

ERCC1 gene or Excision Repair Cross-Complementing Rodent Repair gene which harbored the rs2298881 variant, functions in a nucleotide excision repair pathway [39]. ERCC1 is found to be associated with multiple cancers. ERCC1 variants have also been linked to an increased risk of $\mathrm{BC}$ [40] in women. The variant rs2298881 C > A was found significantly associated with breast cancer. The variant was found to be conferring protection for our $\mathrm{BC}$ in the studied population group. The variant rs2298881 (ERCC1) showed significant expression in the breast with an NES value of -0.260 and a $p$-value of $3.8 \mathrm{e}-9$.

$D C C$ or Deleted in Colorectal Cancer is a gene encoding the netrin 1 receptor. Netrin1 receptor is a transmembrane receptor belonging to the immunoglobulin superfamily. $D C C$ gene is a tumor suppressor gene and is frequently mutated in colorectal carcinomas. $D C C$ is abundantly expressed by neurons and stimulates cell survival and axon regeneration. Apart from mutations in colorectal cancers, studies have highlighted the role of $D C C$ in BC. A variant of the DCC gene, rs2229080, has been found associated with increased BC risk [13]. Our study revealed that rs $2229080 \mathrm{G}>\mathrm{C}$ was significantly associated with breast cancer and the altered allele $\mathrm{C}$ was causing protection in the studied population group.
Though for the variant rs2229080 (DCC) the expression in breast tissue was found non-significant with the NES value of 0.054 with a $p$-value of 0.3 . The positive NES value in rs12190287 (TCF21) is indicative of the upregulation of the expression in the breast tissue and the variants 1,051,266 (SLC19A1) and rs2298881 (ERCC1) with negative NES points towards down-regulated expression in the breast tissue.

The RNA fold analysis revealed the MFE and structural differences in the wild and the altered allele. We also studied the difference in the secondary structures and the MFE values of the wild type allele and the variant allele. There was a decreased MFE in the case of the wild type allele of the variants rs12190287 and rs1051266 providing them an enhanced stable structure than the altered allele. Whereas, the rsIDs rs2229080 and rs2298881 associated with the genes DCC and $E R C C 1$ were found to be causing protection to $\mathrm{BC}$. The MFE values of these variants were lower for the altered allele thus suggesting a more stable structure of these allele variants. The decrease in the MFE of the altered allele points towards an increase in the stability of the secondary structure. These variants have been found to be conferring protection for breast cancer in the studied population. Previously studies have elaborated on the codon selection biasness for a higher negative free energy and folding stability of the RNA secondary structure [10]. Owing to the myriad role of RNA structure in cancer development [11], it might be a potential cancer development risk in the population. Further analysis of the second structure of the genes with the variants highlighted a substantial difference in the MFE and MFE of centroid secondary structure. The differences in the MFE values of the variants have been summarized in the Table S4. The differences in the secondary structures of the alleles have been shown in Fig. 1. On comparing the allele frequencies of the associated allele with 1000genome data, we found substantial differences in the allele frequencies. The differences in the allele frequency of the associated alleles have been depicted in the Fig. S12. An intermediary value of allele frequency for the variant rs1051266 was observed. The allele frequency in the Indian subcontinent comprising of the PJL (Punjabi's from Lahore, Pakistan), ITU (Indian Telugu from the UK) and STU (Sri Lankan Tamil from the UK) was intermediary, around 0.4 in a range of 0 (low) to 1 (high). Similar allele frequencies were observed in the GIH (Gujaratis Indian from Houston, Texas), BEB (Bengali from Bangladesh), GBR (British in England and Scotland), and CEU (Western European Ancestry) populations. The frequency of the variant rs12190287 for found inclined towards a higher side being around 0.7 for the Indian subcontinent. A similar high frequency was seen for the BEB (Bengali from Bangladesh), MXL (Mexican Ancestry from 
Los Angeles USA) and PEL (Peruvians from Lima, Peru) populations. The variant rs2298881 had a comparably lower frequency worldwide. Its frequency in India was on a lower side, around 0.2, with similar frequency observed in BEB (Bengali from Bangladesh) and ASW (Americans of African Ancestry in SW USA) populations. However, in the far eastern populations including the JPT (Japanese in Tokyo, Japan) and CHB (Han Chinese in Beijing, China) a higher frequency of these variants was observed. A very high frequency of about 0.8 for the variant 2,229,080 was observed in the Indian population. A similar high frequency of the variant was observed in JPT (Japanese in Tokyo, Japan) and BEB (Bengali from Bangladesh) populations. Similar frequency rates could indicate a higher $\mathrm{BC}$ rate in these regions. The wide gap between the genetic frameworks of the different populations makes it essential to analyze the genetic heterogeneity among various populations.

\section{Conclusion}

This is the first study that provided the preliminary data for the J\&K population highlighting the role of 15 variants with $\mathrm{BC}$ development. Among the studied variants, four variants namely rs1051266, rs12190287, rs2229080 and rs2298881 showed significant association with $\mathrm{BC}$. The logistic regression with age and BMI revealed the best-fit model to be dominant in the case of rs1051266 (SLC19A1) and rs12190287 (TCF21), while allelic in case of rs2229080 (DCC) and additive in the variant rs2298881 (ERCC1). These variants displayed maximum effect in the populations when incorporated in these models. The RNA fold analysis revealed the structure variations in wild and altered allele along with the differences in the free energy of the secondary structures. In silico analysis of these variants showed the variants to be evolutionarily conserved thus harboring minimum alterations in them over generations, which enables them to maintain their putative structure and function stability efficiently. Three variants rs1051266, rs12190287 and rs2298881 showed significant eQTL effect. The network analysis gave a deeper insight into the nuanced interactions of the candidate genes with other common proteins, alleging a common pathway of function. The associated loci may affect the development of $\mathrm{BC}$ in the women of Jammu and Kashmir and should be further verified in independent data sets. For a better understanding of the gene effect more variants of the genes should be further examined. It might be plausible that there are other variants, which have not been studied and have an eloquent association with BC. Our results provide a clue for further functional validation to reveal underlying genetic mechanisms in BC. These SNPs subsequent to validation can aid in the development of a breast cancer panel specific for the population under study. The specific testing can separate the potential risk targets and early detection could be beneficial in the treatment process. This further can also help in the early detection and personalized medicine for the breast cancer patients.

\section{Supplementary information}

Supplementary information accompanies this paper at https://doi.org/10. 1186/s12885-020-07361-8.

\section{Additional file 1.}

Additional file 2.

\section{Abbreviations}

BMI: Body Mass Index; Cl: Confidence Interval; BC: Breast Cancer; HWE: Hardy Weinberg equilibrium; J\&K: Jammu, and Kashmir; NGS: Next generation sequencing; NES: Normalized effect Size; OR: Odds Ratio; PCR: Polymerase chain reaction; SNP: Single nucleotide polymorphism; SAP: Shrimp alkaline phosphatase; PJL: Punjabi's from Lahore, Pakistan; ITU: Indian Telugu from the UK; STU: Sri Lankan Tamil from the UK; GIH: Gujaratis Indian from Houston, Texas; BEB: Bengali from Bangladesh; GBR: British in England and Scotland; CEU: Western European Ancestry; MXL: Mexican Ancestry from Los Angeles USA; PEL: Peruvians from Lima, Peru; ASW: Americans of African Ancestry in SW USA; JPT: Japanese in Tokyo, Japan; CHB: Han Chinese in Beijing, China

\section{Acknowledgments}

Divya Bakshi would like to acknowledge Dr. Swarkar Sharma, Incharge Massarray facility for his guidance and support.

\section{Authors' contributions}

RK designed the study, DB performed experiments, extrapolated results, and has written the manuscript. DB acknowledges RS, SV, AB and GRB in performing the experimental work, BS in sample collection. AN, VS, IS ran bioinformatic simulations. RS and DA clinically evaluated the patients. DB would like to acknowledge SV and RK in editing the manuscript. The authors have read and approved the manuscript.

Funding

No Funding.

\section{Availability of data and materials}

Data and material are available. The datasets generated or analyzed during the current study are not publicly available but are available with the corresponding author and can be provided on reasonable request.

\section{Ethics approval and consent to participate}

A total of 550 age-matched female samples (150 cases and 400 controls) were recruited for the study. The cases were histo-pathologically confirmed with no evident history of any other cancer. The Institutional Ethical Review Board (IERB) (SMVDU/IERB/18/70) of Shri Mata Vaishno Devi University approved this study. After written informed consent, blood samples, about $2 \mathrm{ml}$ of venous blood was collected into EDTA vacutainers.

Consent for publication

All data is coded, no personal information, images have been used in the study.

\section{Competing interests}

No competing interest.

\section{Author details}

${ }^{1}$ School of Biotechnology, Shri Mata Vaishno Devi University, Katra, Jammu and Kashmir, India. ${ }^{2}$ Ancient DNA Laboratory, Birbal Sahni Institute of Palaeosciences, Lucknow, Uttar Pradesh, India. ${ }^{3}$ Department of Radiotherapy, 
GMC, Kathua, J\&K, India. ${ }^{4}$ Department of Radiotherapy, GMC, Jammu, J\&K, India. ${ }^{5}$ Department of Plant Sciences, Central University of Jammu, Jammu, J\&K, India.

Received: 25 June 2020 Accepted: 31 August 2020

Published online: 07 September 2020

\section{References}

1. Fouad YA, Aanei C. Revisiting the hallmarks of cancer. Am J Cancer Res. 2017;7(5):1016-36

2. Bray F, Ferlay J, Soerjomataram I, Siegel RL, Torre LA, Jemal A. Global cancer statistics 2018: GLOBOCAN estimates of incidence and mortality worldwide for 36 cancers in 185 countries. CA Cancer J Clin. 2018;68(6):394-424.

3. Globocan. India:Globocan 2018. World health organisation; 2018.

4. Qurieshi MA, Khan SM, Masoodi MA, Qurieshi U, Ain Q, Jan Y, et al. Epidemiology of cancers in Kashmir, India: an analysis of hospital data. Adv Prev Med. 2016:2016:1896761.

5. Gravena AAF, Romeiro Lopes TC, Demitto MO, Borghesan DHP, Dell Agnolo CM, Brischiliari SCR, et al. The obesity and the risk of breast cancer among pre and postmenopausal women. Asian Pac J Cancer Prev. 2018;19(9):2429-36.

6. Lobo RA. Hormone-replacement therapy: current thinking. Nat Rev Endocrinol. 2017;13(4):220-31.

7. Surakasula A, Nagarjunapu GC, Raghavaiah KV. A comparative study of preand post-menopausal breast cancer: risk factors, presentation, characteristics and management. J Res Pharm Pract. 2014;3(1):12-8.

8. Sheikh A, Hussain SA, Ghori Q, Naeem N, Fazil A, Giri S, et al. The spectrum of genetic mutations in breast cancer. Asian Pac J Cancer Prev. 2015;16(6): 2177-85.

9. Zhang B, Beeghly-Fadiel A, Long J, Zheng W. Genetic variants associated with breast-cancer risk: comprehensive research synopsis, meta-analysis, and epidemiological evidence. Lancet Oncol. 2011;12(5):477-88.

10. Lilyquist J, Ruddy KJ, Vachon CM, Couch FJ. Common genetic variation and breast cancer risk-past, present, and future. Cancer Epidemiol Biomarkers Prev. 2018;27(4):380-94.

11. Complexo MC, Park DJ, Nguyen-Dumont T, Campbell I, Thompson E, et al COMPLEXO: identifying the missing heritability of breast cancer via next generation collaboration. Breast Cancer Res. 2013:15(3):402.

12. Mahdi KM, Nassiri MR, Nasiri K. Hereditary genes and SNPs associated with breast cancer. Asian Pac J Cancer Prev. 2013;14(6):3403-9.

13. Liu X, Wang X, Fu SW, Wang M, Kang H, Guan H, Zhang S, Ma X, Lin S, Liu K, Feng Y, Dai C, Dai Z. Genetic association of deleted in colorectal carcinoma variants with breast cancer risk: A case-control study. Oncotarget. 2016;7(22):32765-73. https://doi.org/10.18632/oncotarget.9024.

14. Gabriel S, Ziaugra L, Tabbaa D. SNP genotyping using the Sequenom MassARRAY iPLEX platform. In: Current protocols in human genetics; 2009. Chapter 2:Unit 212

15. Sethi I, Sharma V, Sharma I, Singh G, Bhat GR, Bhanwer AJS, et al. Telomere maintenance genes are associated with type 2 diabetes susceptibility in northwest Indian population group. Sci Rep. 2020;10(1):6444.

16. Sharma I, Sharma V, Khan A, Kumar P, Rai E, Bamezai RNK, et al. Ancient human migrations to and through Jammu Kashmir- India were not of males exclusively. Sci Rep. 2018;8(1):851.

17. Purcell S, Neale B, Todd-Brown K, Thomas L, Ferreira MA, Bender D, et al. PLINK: a tool set for whole-genome association and population-based linkage analyses. Am J Hum Genet. 2007;81(3):559-75.

18. Sankoff MZD. RNA secondary structures and their prediction. Bull Mathematical Biol. 1984;46(4):591-621

19. Shannon P, Markiel A, Ozier O, Baliga NS, Wang JT, Ramage D, et al. Cytoscape: a software environment for integrated models of biomolecular interaction networks. Genome Res. 2003;13(11):2498-504.

20. Franz M, Rodriguez H, Lopes C, Zuberi K, Montojo J, Bader GD, et al. GeneMANIA update 2018. Nucleic Acids Res. 2018;46(W1):W60-W4.

21. Clarke L, Zheng-Bradley X, Smith R, Kulesha E, Xiao C, Toneva I, et al. The 1000 genomes project: data management and community access. Nat Methods. 2012;9(5):459-62

22. Machiela MJ, Chanock SJ. LDlink: a web-based application for exploring population-specific haplotype structure and linking correlated alleles of possible functional variants. Bioinformatics. 2015;31(21):3555-7.

23. Wu Y, Dong $X$, Wang $Y$, Wang $Q$, Gu H, Huang W. Association between rs120963, rs152451, rs249935, rs447529, rs8053188, and rs16940342 polymorphisms in the PALB2 gene and breast cancer susceptibility: a metaanalysis. Oncol Res Treat. 2018;41(12):780-6.

24. Shen L, Yin ZH, Wan Y, Zhang Y, Li K, Zhou BS. Association between ATM polymorphisms and cancer risk: a meta-analysis. Mol Biol Rep. 2012;39(5): 5719-25.

25. Yafei Zhang XZ, Liu P, Hong R, Lu H, Ji H, Lu L, et al. Association between FGFR2 (rs2981582, rs2420946 and rs2981578) polymorphism and breast cancer susceptibility: a meta-analysis. Oncotarget. 2016;8(2):3454-70.

26. Chen W, Zhong R, Ming J, Zou L, Zhu B, Lu X, et al. The SLC4A7 variant rs4973768 is associated with breast cancer risk: evidence from a case-control study and a meta-analysis. Breast Cancer Res Treat. 2012;136(3):847-57.

27. Alborzi A, Houshmand M, Hosseini M. ER and PR Positive, or Her2 Negative Tumor of rs2363956 and rs3803662 GWAS in Breast. Cancer, Gene Cell Tissue. 2017;4(4):e63407. https://doi.org/10.5812/gct.63407.

28. Johansson H, Aristarco V, Gandini S, Gjerde J, Macis D, Guerrieri-Gonzaga A, et al. Prognostic impact of genetic variants of CYP19A1 and UGT2B17 in a randomized trial for endocrine-responsive postmenopausal breast cancer. Pharmacogenomics J. 2020;20(1):19-26.

29. Hui Li YX, Mei H, Peng L, Li X, Tang J. The TERT rs 2736100 polymorphism increases cancer risk: a meta-analysis. Oncotarget. 2017:8(24):38693-705.

30. Yang H, Li J, Tang R, Liu Y, Shao Y, Huang Q, et al. Telomere reverse transcriptase (TERT) rs2735940 increases cancer risk. Med Sci Monit. 2015;21: 612-6.

31. Yin $\mathrm{H}$, Hardikar S, Lindstroem S, Hsu L, Anderson KE, Banbury BL, et al. Telomere maintenance variants and survival after colorectal cancer: smoking- and sex-specific associations. Cancer Epidemiol Biomarkers Prev. 2020;29(9):1824. https://doi.org/10.1158/1055-9965.EPI-19-1507.

32. Shi J, Tong J, Cai S, Qu X, Liu Y. Correlation of the BACH1 Pro919Ser polymorphism with breast cancer risk: a literature-based meta-analysis and meta-regression analysis. Exp Ther Med. 2013;6(2):435-44.

33. Morales-Santana S, Morell S, Leon J, Carazo-Gallego A, Jimenez-Lopez JC, Morell M. An overview of the polymorphisms of circadian genes associated with endocrine cancer. Front Endocrinol (Lausanne). 2019:10:104.

34. Zhang ZY, Xuan Y, Jin XY, Tian X, Wu R. CASP-9 gene functional polymorphisms and cancer risk: a large-scale association study plus metaanalysis. Genet Mol Res. 2013;12(3):3070-8.

35. Naushad SM, Divya C, Janaki Ramaiah M, Hussain T, Alrokayan SA, Kutala VK Population-level diversity in the association of genetic polymorphisms of one-carbon metabolism with breast cancer risk. J Community Genet. 2016; 7(4):279-90.

36. Gong Z, Yao S, Zirpoli G, David Cheng TY, Roberts M, Khoury T, et al. Genetic variants in one-carbon metabolism genes and breast cancer risk in European American and African American women. Int J Cancer. 2015;137(3): 666-77.

37. Gao $X$, Yang J, Wang M, Zhang J. TCF21 genetic polymorphisms and breast cancer risk in Chinese women. Oncotarget. 2016;7(34):55757-64. https://doi. org/10.18632/oncotarget.9825.

38. Wang J, Gao X, Wang M, Zhang J. Clinicopathological significance and biological role of TCF21 mRNA in breast cancer. Tumour Biol. 2015;36(11): 8679-83

39. Formica V, Doldo E, Antonetti FR, Nardecchia A, Ferroni P, Riondino S, et al. Biological and predictive role of ERCC1 polymorphisms in cancer. Crit Rev Oncol Hematol. 2017;111:133-43.

40. Zhao R, Ying MF. Association between ERCC1 and ERCC2 polymorphisms and breast cancer risk in a Chinese population. Genet Mol Res. 2016;15(1): 15017263

\section{Publisher's Note}

Springer Nature remains neutral with regard to jurisdictional claims in published maps and institutional affiliations. 